\title{
Defining the frontiers between antifungal resistance, tolerance and the concept of persistence
}

\author{
Eric Delarze, Dominique Sanglard* \\ Institute of Microbiology, University Hospital Lausanne and University Hospital Center, Rue de Bugnon 48, CH-1011 Lausanne, Switzerland
}

\section{A R T I C L E I N F O}

\section{Keywords:}

Antifungal resistance

Tolerance

Persistence

\begin{abstract}
A B S T R A C T
A restricted number of antifungal agents are available for the therapy of fungal diseases. With the introduction of epidemiological cut-off values for each agent in important fungal pathogens based on the distribution of minimal inhibitory concentration (MIC), the distinction between wild type and drugresistant populations has been facilitated. Antifungal resistance has been described for all currently available antifungal agents in several pathogens and most of the associated resistance mechanisms have been deciphered at the molecular level. Clinical breakpoints for some agents have been proposed and can have predictive value for the success or failure of therapy. Tolerance to antifungals has been a much more ignored area. By definition, tolerance operates at antifungal concentrations above individual intrinsic inhibitory values. Important is that tolerance to antifungal agents favours the emergence of persister cells, which are able to survive antifungal therapy and can cause relapses. Here we will review the current knowledge on antifungal tolerance, its potential mechanisms and also evaluate the role of antifungal tolerance in the efficacy of drug treatments.
\end{abstract}

(C) 2015 Elsevier Ltd. All rights reserved.

\section{Introduction}

Resistance of microbes to anti-infectives is an increasing threat to human health and has a significant socio-economic impact in national health systems. However, the actions undertaken to counteract drug resistance remain largely insufficient. Bacterial antibiotic resistance is a dominant research area due the high prevalence of hospital bacterial infections. Resistance to antifungal drugs has received less attention, although the occurrence of fungal diseases is far from negligible. These diseases range from superficial (oral or genito-urinary tract thrushes) to life-threatening systemic infections and cause high levels of morbidity and mortality. It has been reported that about 1.7 billion people worldwide are infected with pathogenic fungi, from which 1.5 million die every year (Brown et al., 2012). While the majority of pathogenic fungi are opportunistic pathogens, they become deadly pathogens in immunocompromised patients. The increased use of immunosuppressive therapies for cancer and organ transplants has resulted in rising incidence of invasive fungal diseases. The number of fungal sepsis has risen by 207\% between 1979 and 2000 in the United States and disseminated systemic candidiasis is associated with alarmingly high rates of mortality of up to 50\% (Martin et al., 2003).

\footnotetext{
* Corresponding author. Tel.: +41213144083.

E-mail address: Dominique.sanglard@chuv.ch (D. Sanglard).
}

Candida albicans remains a major fungal pathogen in invasive diseases and is followed by several Candida spp. including C. glabrata, C. parapsilosis and C. tropicalis (Arendrup, 2010). Among filamentous fungi, Aspergilli spp. are predominant and especially A. fumigatus. This fungal species is a major cause of death in acute leukemia patients. Approximately $30 \%$ of bone marrow or organ transplants will develop invasive aspergillosis with poor prognosis (Richardson and Lass-Flörl, 2008).

A restricted number of chemical classes are currently in clinical use as antifungal agents including polyenes, pyrimidine analogues, echinocandins and triazoles. Polyenes such as amphotericin $B$ have the ability to bind ergosterol and act as a sterol "sponge" thus destabilizing membrane functions (Anderson et al., 2014). Amphotericin $B$ exerts intrinsic toxic effects in human, however this negative effect can be alleviated by liposome formulations (Sanglard and Odds, 2002). Echinocandins block the catalytic subunit of the $ß-1,3$ glucan synthase and thus inhibit cell wall biosynthesis (Arendrup and Perlin, 2014). Triazoles are still the mostly used antifungals. These compounds target a specific step in ergosterol biosynthesis catalysed by lanosterol $14 \alpha$-demethylase (Sanglard and Odds, 2002). Fluconazole is the major triazole in clinical settings, probably due to its high oral availability and tolerability by patients.

The activity of these different antifungal classes on fungal pathogens can vary and several factors are involved in this process. Variations of antifungal activities have different bases, including occurrence of intrinsic or acquired resistance. Antifungal activities 
can also depend on underlying mechanisms that are related to antifungal tolerance. Finally, antifungal activity can also be altered by persistence mechanisms. The terminology and definitions behind these different mechanisms is often not properly used in published studies. The terms "drug resistance" and "drug tolerance" are often used for reporting the same phenotypes, thus creating confusions. In this review, we will attempt to more clearly define these different terms and summarize the present knowledge on the different mechanisms that determine the activity of antifungal agents. We will also evaluate the impact of antifungal tolerance on the fate of antifungal therapy.

\section{What is understood by antifungal activity?}

There are two basic antifungal susceptibility patterns in vitro among fungal pathogens. Either the presence of the antifungal drug results in a decrease or absence of growth capacity at a given concentration as compared to untreated control or the antifungal drug does not affect fungal growth of specific species at any concentrations. The absence of drug activity in a species that was not pre-exposed to the tested agent is also known as intrinsic resistance. For example, it is known that wild type $C$. albicans is susceptible to fluconazole, whereas A. fumigatus is intrinsically resistant to this azole (Pfaller, 2012). Several other examples of intrinsic resistance are given in Table 1. Antifungal activity is usually measured with standard broth dilution protocols enabling inter-laboratory comparisons. Two major protocols are currently used, either originating from two major antifungal susceptibility testing subcommittees (CLSI: Clinical Laboratory Standards Institute; EUCAST: European Committee on Antimicrobial Susceptibility Testing). The protocols yield so-called minimal inhibition concentration (MIC) values (given in $\mu \mathrm{g} / \mathrm{ml}$ ) as measures of antifungal activity. These protocols use microtitre 96 well plates, albeit with well formats that are specific for each method. While these protocols differ in several technical aspects, the agreement between the two methods in terms of antifungal activities is generally high. For example, MIC values for isavuconazole in $C$. albicans range from $\leq$
0.008 to $0.03 \mu \mathrm{g} / \mathrm{ml}$ for CLSI while these values range from $\leq 0.008$ to $0.015 \mu \mathrm{g} / \mathrm{ml}$ for EUCAST (Pfaller et al., 2013). Variability in agreements depends more on the tested fungal species (Arendrup et al., 2013; Rambach et al., 2011). One of the major differences between the two methods is their incubation time (24- and $48 \mathrm{~h}$ for CLSI; $24 \mathrm{~h}$ for EUCAST) before measurement of MIC values. Second, the MIC values are determined either by optical density (OD) measurements with EUCAST (antifungal concentration that results in $\leq 50 \%$ of decrease in OD as compared to control) or by visual inspection with CLSI.

\section{What is antifungal resistance?}

Now that antifungal activity can be measured by specific protocols, it is possible to determine how these activities are distributed within a same fungal species and how these activities can be compared with other species. When taking the distribution of fluconazole MICs in C. albicans clinical isolates, a bell-shaped Gaussian distribution can be observed (Fig. 1). Such distributions can be obtained with other antifungal agents and other fungal species (Arendrup et al., 2013). MIC distributions can identify isolates that are different from wild type isolates. The EUCAST defines the limit above which non-wild type isolates can be detected as the epidemiological cut off (ECOFF) value. The ECOFF value is defined as the upper limit of the wild type population (Fig. 1) and can be selected based on visual inspection (the eye ball method). Taking Fig. 1 as an example, the ECOFF value for fluconazole in C. albicans is $1 \mu \mathrm{g} / \mathrm{ml}$. Above this value, isolates differ from the wild type population by the potential occurrence of resistance mechanism. Thus, ECOFF values help to identity non-wild type isolates exhibiting potential resistance mechanisms to a given drug within a given population. Table 2 summarizes these values for several agents and major fungal pathogens.

Considering that antifungal resistance occurs in vitro above the ECOFF values, it should be possible to identify the resistance mechanisms prevailing in non-wild type isolates. With the help of antifungal resistance mechanisms known for C. albicans and azoles

Table 1

Overview on the activity of antifungal agents in several fungal pathogens ${ }^{\mathrm{a}}$.

\begin{tabular}{|c|c|c|c|c|c|c|c|c|c|}
\hline \multirow[t]{2}{*}{ Organism } & \multicolumn{9}{|l|}{ Antifungal agent ${ }^{\mathrm{b}}$} \\
\hline & Amphotericin B & Fluconazole & Itraconazole & Voriconazole & Posaconazole & Anidulafungin & Caspofungin & Micafungin & Flucytosine \\
\hline \multicolumn{10}{|l|}{ Aspergillus species } \\
\hline A. flavus & \pm & - & + & + & + & + & + & + & - \\
\hline A. fumigatus & + & - & + & + & + & + & + & + & - \\
\hline A. niger & + & - & + & + & + & + & + & + & - \\
\hline A. terreus & - & - & + & + & + & + & + & + & - \\
\hline \multicolumn{10}{|l|}{ Candida species } \\
\hline C. albicans & + & + & + & + & + & + & + & + & \pm \\
\hline C. glabrata & + & \pm & \pm & \pm & \pm & + & + & + & \pm \\
\hline C. krusei & + & - & \pm & + & + & + & + & + & - \\
\hline C. lusitaniae & - & + & + & + & + & + & + & + & + \\
\hline C. parapsilosis & + & + & + & + & + & \pm & \pm & \pm & + \\
\hline C. tropicalis & + & + & + & + & + & + & + & + & + \\
\hline \multicolumn{10}{|l|}{ Other species } \\
\hline Cryptococcus neoformans & + & + & + & + & + & - & - & - & + \\
\hline Coccidioides species & + & + & + & + & + & \pm & \pm & \pm & - \\
\hline Blastomyces & + & + & + & + & + & \pm & \pm & \pm & - \\
\hline Histoplasma species & + & + & + & + & + & \pm & \pm & \pm & - \\
\hline Fusarium species & \pm & - & - & \pm & \pm & - & - & - & - \\
\hline Scedosporium apiospermum & \pm & - & \pm & \pm & + & $\pm^{\mathrm{c}}$ & $\pm^{\mathrm{c}}$ & $\pm^{\mathrm{c}}$ & - \\
\hline Scedosporium prolificans & - & - & - & \pm & \pm & - & - & - & - \\
\hline Zygomycetes & \pm & - & - & - & \pm & - & - & - & - \\
\hline
\end{tabular}

a Adapted from Ashley et al. (2006), Espinel-Ingroff (2003) and Denning and Hope (2010).

b Plus signs (+) indicate that the antifungal agent has activity against the specified organism. Minus signs (-) indicate that the antifungal agent does not have activity against the specified organism. Plus-minus signs $( \pm)$ indicate that the agent has different activities $(+$ and -$)$ against the specified organism.

c Adapted from data provided by Lackner et al. (2012) and Castanheira et al. (2012). 


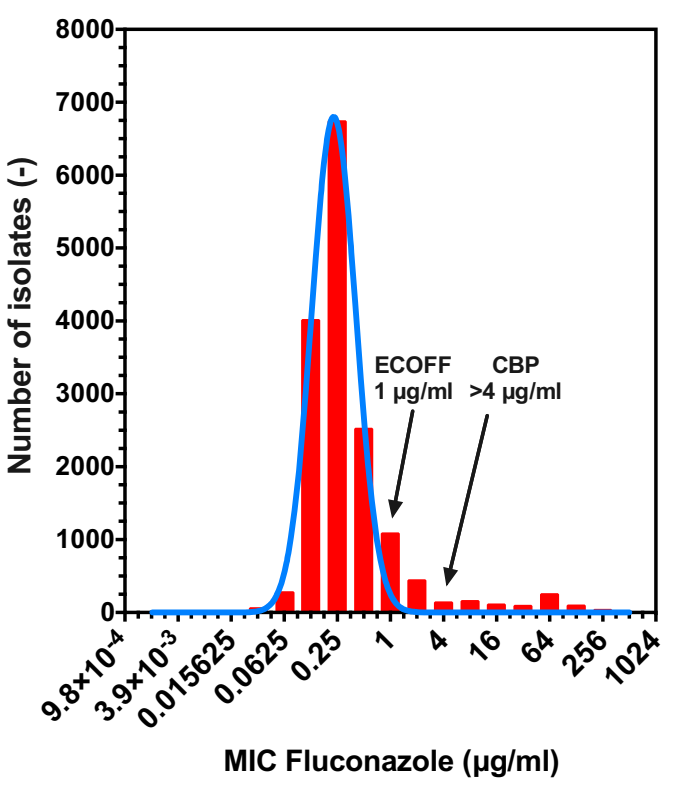

Fig. 1. Distribution of fluconazole MICs in C. albicans. MIC data were obtained from the EUCAST MIC Database (http://mic.eucast.org/Eucast2/SearchController/). The bell shaped blue curve was obtained with GraphPad Prism with Log Gaussian curve fitting. Epidemiological cut off (ECOFF) and clinical breakpoint for resistance (CBP) are indicated with corresponding values.

(see for review Sanglard (2011)), we observed that, from a total of 38 isolates, 25 contained azole resistance mechanisms investigated at the molecular level and only 1 isolate without known resistance mechanism was above the ECOFF value threshold (Fig. 2). Three isolates with known resistance mechanism had fluconazole MICs below this threshold. Interestingly, these three isolates contain each a single resistance mechanism (amino acid substitution in Erg11p) with minor effect on fluconazole MICs, which indicates that some specific azole resistance mutations might not be correctly identified by the established ECOFF threshold. Taken together, these data however strongly support the notion that the ECOFF value can distinguish between wild type and non-wild type isolates.

Clinical breakpoints (CBP) should reflect the relationship between in vitro antifungal activity and the likelihood of success or failure of therapy of a given drug. These clinical breakpoints were established by EUCAST and CLSI using several clinical parameters including in vivo PK-PD, resistance mechanisms and clinical response (Table 2). Referring to Fig. 1, the EUCAST CBP for

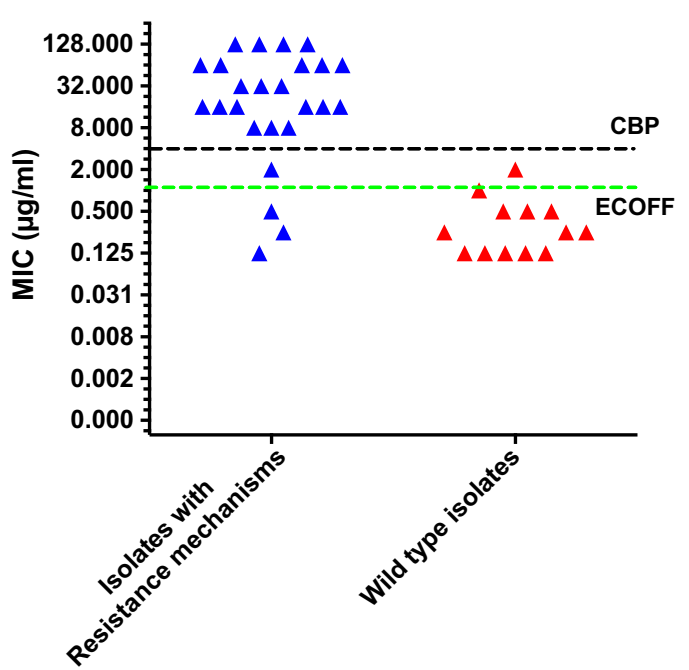

Fig. 2. Distribution of fluconazole MICs in C. albicans according to occurrence of azole resistance mechanisms. Data were obtained from data published in (Coste et al., 2007, 2009). CBP for resistance and ECOFF values are indicated by dotted black and green lines, respectively. Blue and red triangles indicate MIC values from isolates with and without known resistance mechanisms, respectively.

fluconazole in C. albicans categorizes all isolates as azole-resistant. The isolates exhibit known azole resistance mechanisms and one could expect that they would be refractory to azole therapy. However, CBP can have limited predictive power. For example, while a $92 \%$ success rate for $C$. albicans 550 events were observed with fluconazole MICs of $\leq 2 \mu \mathrm{g} / \mathrm{ml}$, it decreases to $83 \%$ success among 52 events with MICs of $\geq 4 \mu \mathrm{g} / \mathrm{ml}$ and drops to $37 \%$ success among 212 events with MIC of $8 \mu \mathrm{g} / \mathrm{ml}$ (Pfaller, 2012). From a fungal point of view, the relative lack of reliability of the CBP for predicting patient outcome could be explained by the fact that antifungal susceptibility methods have a limited capacity to predict the response of fungal pathogens to antifungals in vivo.

\section{What is antifungal tolerance?}

Among the diverse characteristics contributing to the efficacy of a given drug, an important and often neglected parameter is the ability of fungal pathogens to tolerate the presence of a drug. Drug tolerance is defined in the field of antimicrobial agents as the ability to withstand killing at drug concentrations above the MIC (Fridman et al., 2014). Antibiotic tolerance is reversible and thus is rather the result of epigenetics as opposed to drug resistance which depends

Table 2

ECOFF and CBP of different antifungal agents and fungal species ${ }^{\mathrm{a}}$.

\begin{tabular}{|c|c|c|c|c|c|c|c|c|c|c|}
\hline \multirow{3}{*}{ Species } & \multirow{3}{*}{ Method } & \multicolumn{3}{|c|}{ ECOFF $(\mu \mathrm{g} / \mathrm{ml})$} & \multicolumn{6}{|c|}{$\mathrm{CBP}(\mu \mathrm{g} / \mathrm{ml})$} \\
\hline & & \multirow[t]{2}{*}{ Fluconazole } & \multirow[t]{2}{*}{ Anidulafungin } & \multirow[t]{2}{*}{ Micafungin } & \multicolumn{2}{|c|}{ Fluconazole } & \multicolumn{2}{|c|}{ Anidulafungin } & \multicolumn{2}{|c|}{ Micafungin } \\
\hline & & & & & $S^{b}$ & $R^{\mathrm{b}}$ & $S$ & $R$ & $S$ & $R$ \\
\hline \multirow[t]{2}{*}{ C. albicans } & CLSI & 0.5 & $\leq 0.12$ & $\leq 0.03$ & 2 & 4 & 0.25 & 0.5 & 0.25 & 0.5 \\
\hline & EUCAST & 1 & 0.03 & 0.015 & 2 & 4 & 0.03 & 0.03 & 0.016 & 0.016 \\
\hline \multirow[t]{2}{*}{ C. glabrata } & CLSI & 32 & $\leq 0.25$ & $\leq 0.03$ & 0.002 & 32 & 0.12 & 0.25 & 0.06 & 0.12 \\
\hline & EUCAST & 32 & 0.06 & 0.03 & 0.002 & 32 & 0.06 & 0.06 & 0.03 & 0.03 \\
\hline \multirow[t]{2}{*}{ C. parapsilosis } & CLSI & 2 & $\leq 4$ & $\leq 4$ & 2 & 4 & 2 & 4 & 2 & 4 \\
\hline & EUCAST & 2 & 4 & 2 & 2 & 4 & 0.002 & 4 & 0.002 & 2 \\
\hline \multirow[t]{2}{*}{ C. tropicalis } & CLSI & 2 & $\leq 0.12$ & $\leq 0.12$ & 2 & 4 & 0.25 & 0.5 & 0.25 & 0.5 \\
\hline & EUCAST & 2 & 0.06 & 0.06 & 2 & 4 & 0.06 & 0.06 & $\mathrm{IE}^{\mathrm{d}}$ & IE \\
\hline \multirow[t]{2}{*}{ C. krusei } & CLSI & 64 & $\leq 0.12$ & $\leq 0.12$ & $-{ }^{c}$ & - & 0.25 & 0.5 & 0.25 & 0.5 \\
\hline & EUCAST & 128 & 0.06 & 0.25 & - & - & 0.06 & 0.06 & IE & IE \\
\hline
\end{tabular}

\footnotetext{
a Data obtained from published studies (Arendrup et al., 2013; Maubon et al., 2014; Pfaller et al., 2010, 2014).

b Categorical discrimination between resistant $(R)$ and susceptible $(S)$.

c “-" Indicates that susceptibility testing is not recommended as the species is a poor target for therapy with the drug.

d "IE" indicates that there is insufficient evidence that the species in question is a good target for therapy with the drug.
} 

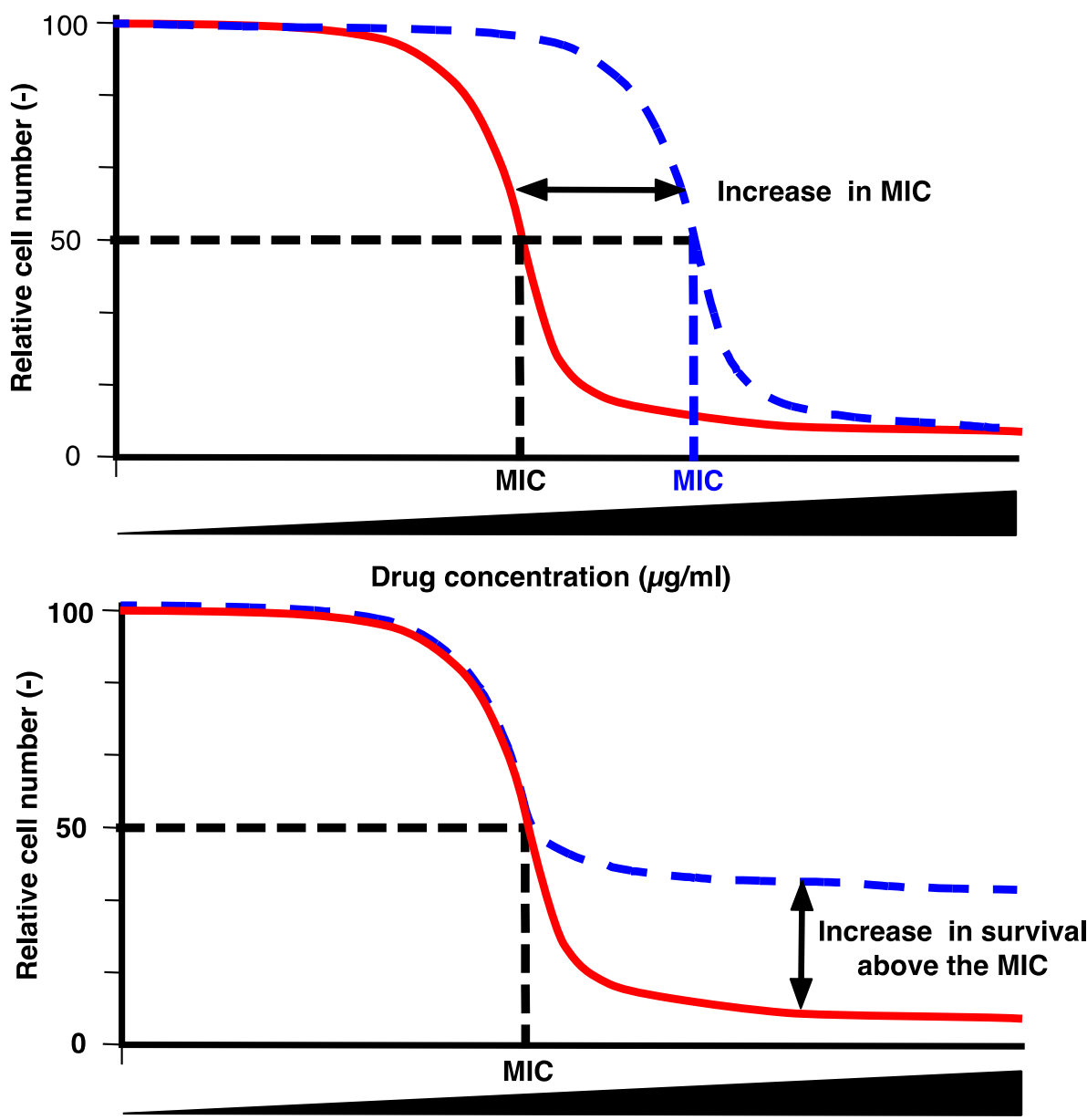

Drug concentration $(\mu \mathrm{g} / \mathrm{ml})$

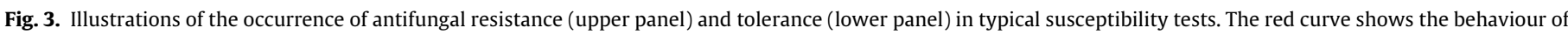

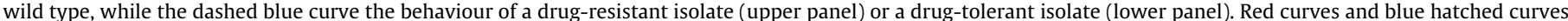
correspond to data of wild-type and drug-resistant (upper panel) or drug-tolerant (lower panel) isolates, respectively.

on genes and their mutations. Drug tolerance can result in the presence of so-called persisters, which are dormant cells appearing stochastically in the presence of antibiotics (Lewis, 2007). Drug tolerance is a concept that was proposed with antibacterial drugs possessing a cidal effect. Tolerant persisters can therefore be simply distinguished by their ability to withstand antibiotic killing above the MIC. The presence of persisters in bacterial biofilms and indeed the presence of persister cells in antibiotic treatments might be important in the aetiology of many recalcitrant infectious diseases (Lewis, 2007). Recent data show that persister bacterial cells are favoured by nutrient starvation and mechanisms that induce decrease in membrane potential, thus resulting in a dormant stage (Verstraeten et al., 2015).

In line with what has been published in bacteria, antifungal tolerance could be also defined as the ability of cells to survive at drug concentrations that exceed the MIC. In this sense, antifungal tolerance is different from antifungal resistance, which itself reflects an increase of MIC value independent of the ability to survive at drug concentrations higher than this value (Fig. 3). On the other hand, antifungal tolerance needs to be adjusted to antifungal drug classes containing either fungistatic (azoles) or fungicidal drugs (candins, amphotericin B). Basically, it is possible to say that fungal pathogens are generally tolerant to azoles, since fungal cells are not killed by drug concentrations above individual MICs. However, and this is a specificity of azoles, the degree of azole tolerance can vary within a same fungal species. The degree of azole tolerance reflects a phenomenon that is known as the "trailing growth" in susceptibility tests. Trailing growth of specific Candida spp. with azoles has been repeatedly reported and describes residual growth of these species in the presence of azoles at concentrations above the MIC (Marchetti et al., 2000b; Marr et al., 1999). Trailing growth can be more or less pronounced and mirrors the degree of azole tolerance. Fig. 4 illustrates the different degrees of tolerance to fluconazole

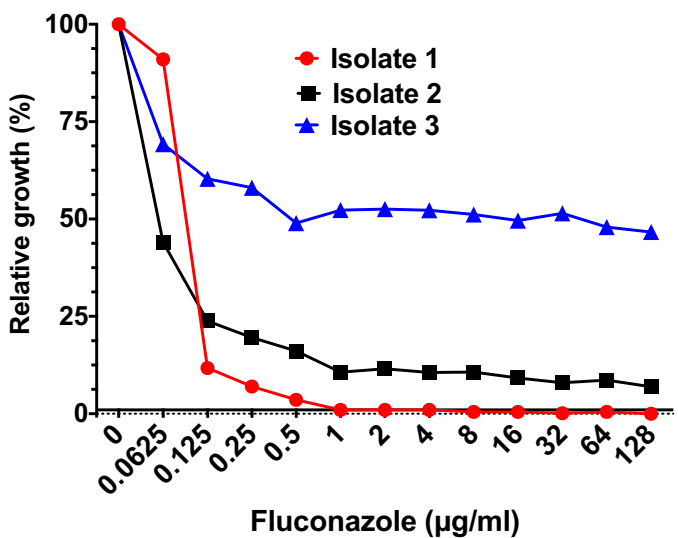

Fig. 4. MIC susceptibility test illustrating the different degrees of antifungal tolerance. 
in several $C$. albicans isolates. Isolate 3 shows the highest degree of azole tolerance with a MIC at $0.5 \mu \mathrm{g} / \mathrm{ml}$. Isolate 1 and 2 exhibit lower degrees of azole tolerance as compared to isolate 3 and have both fluconazole MIC values between 0.0625 and $0.125 \mu \mathrm{g} / \mathrm{ml}$. Taking larger subsets of clinical isolates with low fluconazole MICs, our own data show that the majority resembles profiles of isolates 1 and 2 (D. Sanglard, unpublished). Tolerance with fungicidal agents such as caspofungin or amphotericin B can be estimated by measuring cell survival in MIC tests. For example, after $24 \mathrm{~h}$ exposure of two $C$. glabrata isolates (\#4205 and \#4393) to caspofungin concentration 4 times above the MIC, cell survival was decreased as compared to the incoculum in both isolates, however one isolate (\#4393) survived by 2 logs CFU better than the other. Thus, isolate \#4393 can be considered as more tolerant to caspofungin than \#4205 (Barchiesi et al., 2005).

C. albicans biofilms are known to be resistant to several antifungal agents. Contrary to resistance in planktonic cells, which can acquire resistance by genetic modifications, resistance of $C$. albicans biofilm cells is only a transient state. Addressing whether or not biofilms cells are resistant or tolerant to antifungal agents challenges the definition of resistance and tolerance. Taking as example the MIC of amphotericin B in planktonic and biofilm conditions, it has been reported that the MIC of biofilm cells was 16-fold higher than planktonic cells, thus implying that biofilm formation results in amphotericin B resistance (Tobudic et al., 2010). Above this MIC value, biofilm cells are able to survive in the presence of amphotericin B, as indicated by a recent study (Li et al., 2015a). When admitting that drug tolerance is the ability to survive at drug concentration higher than the MIC, these surviving cells are tolerant to amphotericin B. These surviving cells can be defined as persister cells and thus persisters originate from drug tolerance mechanisms.

\section{The occurrence of fungal persitence}

Success of antifungal therapy could be considered as the absence of disease symptoms when therapy resumes. Endpoints quantifying the success of a therapy in animal assays are often associated with fungal burdens in tissues and reduction of these burdens as compared to untreated animals. Pharmacokinetic (PK) and pharmacodynamic (PD) parameters have been proposed to reach the optimal dosage which could predict the success of antifungal therapy. Animal model studies have determined that the ratio of the $24 \mathrm{~h}$ area under the concentration-time curve (AUC) divided by the $\mathrm{MIC}$ (AUC/MIC) is a PD parameter that correlates best with fluconazole efficacy. Ratios of AUC/MIC between 20 and 50 can predict a successful therapy (Lepak and Andes, 2014). Clinical data are less clear about the validity of these PD parameters. Recent data for fluconazole suggest that a ratio $>400$ could be more accurate to predict positive outcomes for C. albicans infections (Brosh-Nissimov and Ben-Ami, 2015). CBP have been also proposed which could help predicting the outcomes of antifungal treatments (Hope and Drusano, 2009). Nevertheless, even if a therapy is considered as successful, the fungal pathogen (a persister) may not be totally eradicated from host tissues. In this case, if antifungal therapy is abandoned, relapse of infection could occur. In animal models of fungal infections, this aspect is neglected or not addressed in the vast majority of reports. However, infection relapses are relevant in clinical settings. Infection relapses may be caused by fungal persisters, which are cells able to survive in the host environment even after antifungal therapy. These cells are present in small numbers and can be hidden from detection in infected tissues. A recent illustrative example from persister cells has been given by Jacobsen et al. (2014) when caspofungin was administered in an invasive murine model of $C$. albicans infection. The authors used in vivo imaging techniques to reveal that a significant portion of $C$. albicans cells were still present in the gall bladder of mice treated with this agent. The in vivo imaging technique was crucial to enable the detection of bioluminescent $C$. albicans cells in this specific organ, which would have been probably missed by conventional detection methods. Interestingly Jacobsen et al. (2014) discovered that bile acids present in the gall bladder were counteracting the activity of several antifungal agents including candins, azoles and amphotericin $B$. Thus, the gall bladder could provide an optimal environment for the occurrence of persisters in C. albicans. The basis for the formation of persisters is still unknown. However, persisters may form from increased tolerance to antifungal agents, as earlier mentioned. As in the case of tolerance, persistence is also a reversible state and thus differs from the occurrence of resistance which is mediated by stable gene alterations.

Persister cells are also known as phenotypic variants of wild type cells arising in biofilms (LaFleur et al., 2006). These cells are in a dormant state in analogy with bacterial persisters. Whether the biofilms persisters and those occurring in host tissues during antifungal therapy are cells with similar features in still unknown. A recent study has explored the tolerance mechanisms of the persisters formed in a biofilm and exposed to an amphotericin B concentration above the MIC (Li et al., 2015a). The authors used proteomic profiles of $C$. albicans biofilm persister fractions. Enzymes crucial in glycolysis, tricarboxylic acid cycle and protein synthesis were markedly decreased as compared to untreated cells, indicating that major metabolic activities are kept at low levels in persisters. The current findings suggest that a subtle metabolic control plays a role in the survival and antifungal tolerance of $C$. albicans biofilm persisters.

\section{Mechanisms of antifungal tolerance}

Tolerance mechanisms to antifungal drug have received little attention as compared to the study of resistance mechanisms. One of the approaches to resolve the basis for antifungal tolerance was by the search of substances which will inhibit tolerance. Loss of tolerance to azoles was first demonstrated when cyclosporine A(CsA), an inhibitor of calcineurin activity, was added simultaneously to fluconazole in MIC checkerboard tests in C. albicans (Marchetti et al., 2000b; Sanglard et al., 2003). The construction of mutants lacking the gene $C N A / C M P 1$ gene encoding calcineurin subunit A established that azole tolerance was mediated by calcineurin activity and its calcium-dependent activation pathway (Sanglard et al., 2003). Interestingly, tolerance to other inhibitors including caspofungin was also decreased in the $c n a \Delta / \Delta$ mutant as compared to wild type, suggesting that calcineurin plays an important role in the response of $C$. albicans to other stress generating agents (Sanglard et al., 2003). It is important to underscore that calcineurin regulates drug tolerance only and not drug resistance, since the presence or absence of this activity does not alter drug susceptibility as measured by drug susceptibility assays (Sanglard et al., 2003). Calcineurin was also shown as a client protein of the chaperone Hsp90. Inhibition of Hsp90 functions is comparable to a loss of calcineurin activity and therefore mimic a calcineurin mutant with respect to drug tolerance (Cowen, 2013). It has also been shown that Hsp90 stabilizes a terminal kinase of the PKC pathway and this may participate to antifungal tolerance (LaFayette et al., 2010). Additionally, Hsp90 has a buffering capacity when living cells are exposed to various insults. In yeast, Hsp90 can therefore determine the fate and trajectory of antifungal resistance, a concept that was addressed and verified in S. cerevisiae (Cowen and Lindquist, 2005). Of interest is that Hsp90 was shown to undergo acetylation/deacetylation cycles and that the state of acetylation in C. albicans could determine Hsp90 activity and chaperone activity on calcineurin (Robbins et al., 2012). Inhibiting deacetylase activity was shown to reduce 
azole trailing growth (i.e. azole tolerance) in C. albicans, thus being consistent with the effect of acetylation on Hsp90 and its client protein calcineurin (Li et al., 2015b).

Tetracycline and its derivatives, which are antibiotics inhibiting protein synthesis, were found to potentiate the activity of fluconazole in C. albicans. For example, minocycline is synergistic with fluconazole against fluconazole-resistant $C$. albicans isolates (Liu et al., 2014). Synergism between doxycycline (DOX), another tetracycline derivative, and fluconazole has also been reported in azole-resistant isolates (Gao et al., 2013). This conclusion was also reported by Fiori and van Dijck (2012) when high DOX concentrations were used $(>200 \mu \mathrm{g} / \mathrm{ml})$. This study also revealed that DOX reduced tolerance of $C$. albicans to fluconazole. This effect was dependent on iron availability in the culture medium, which could be modulated by the iron-chelating ability of DOX. Interestingly, DOX had a fungicidal effect at fluconazole concentrations above the MIC. Taken together, these results suggest a relationship between iron homeostasis and antifungal tolerance.

Antifungal tolerance mechanisms can also be approached by screening of yeast mutants in which azoles have a fungicidal effect. For example, it was showed that a $C$. albicans mutant lacking the transcriptional activator UPC2 was killed by fluconazole as opposed to wild type (Vasicek et al., 2014). UPC2 regulates sterol homeostasis in C. albicans and since azoles perturb this pathway, one can expect dramatic effects for the fungus. The study of Vasicek et al. (2014) showed that the UPC2 mutant became hypersusceptible to azoles as measured by a MIC decrease as compared to wild type. UPC2 therefore confounds effects on both azole tolerance and resistance. A similar study but performed with $C$. glabrata mutants identified the calcium channels genes $\mathrm{CCH} 1$ and MID1 as critical for fluconazole tolerance, since absence of these genes converted this azole into a fungicidal agent (Kaur et al., 2004). These data therefore suggest a connection between calcium entry and azole tolerance. This may be expected since a functional calcium homeostasis is required for calcineurin activity, which itself is important for azole tolerance as mentioned above.

In another recent study, it was shown that a $C$. albicans $v p s 21 \Delta / \Delta$ mutant which is blocked in membrane trafficking through the late endosomal prevacuolar compartment, was able to grow significantly more than the wild type in the presence of fluconazole under standard susceptibility testing conditions. This growth behaviour resembled characteristics of trailing. Thus, VPS21 might be a mediator of azole tolerance, however this is rather an absence of its activity that favours trailing. The authors of the study speculated that vacuole biogenesis and sterol homeostasis may be connected and that toxic sterols produced upon azole treatment are redistributed to different cell compartments, which is less toxic for the fungus (Luna-Tapia et al., 2015).

Lastly, Marr et al. (1999) showed that azole tolerance in $C$. albicans may be dependent on $\mathrm{pH}$ of the culture medium. Measuring azole MIC at neutral $\mathrm{pH}$ resulted in trailing growth at azole concentration above the MIC. This phenotype was not observed at low $\mathrm{pH}$. These results suggest that the $\mathrm{pH}$ may modulate azole tolerance. However, the study of Marr et al. (1999) used two different medium compositions in which the $\mathrm{pH}$ was also different. While the $\mathrm{pH}$ hypothesis in azole tolerance is attractive, further experiments need to be performed in a medium-independent manner.

Tolerance mechanisms to antifungal classes different than azoles are still poorly understood. As mentioned above the calcineurin/HSP90 complex is critical for tolerance of $C$. albicans to echinocandins. While echinocandins have intrinsic fungicidal activities in C. albicans, compromising calcineurin and/or HSP90 activity increases to variable extent this fungicidal activity. This is consistent with a role of calcineurin/HSP90 in echinocandin tolerance. The calcineurin/HSP90 complex appears also important for tolerance to a wide range of growth inhibitors not only in $C$. albicans but also in other fungal species. One issue in the identification of novel mediators of tolerance of non-azole antifungals is that a high number of studies report antifungal susceptibility assays which cannot predict and measure drug tolerance. In many instances, serial dilutions on agar plates containing a given drug and a given fungal isolate are performed and can reflect enhanced or decreased drug susceptibility. In order to assess drug tolerance, cell viability assays should be performed which is mostly not the case. In some other cases, antifungal tolerance was correctly addressed. For example, in a study published by Miyazaki et al. (2010), C. glabrata was subjected to time-kill assays in the presence of micafungin. A $C$. glabrata mutant lacking the protein mitogen-activated kinase gene SLT2 was subjected to the same assay, however this mutant was killed 10-times more efficiently than the parent wild type as the same supra-MIC drug concentration. The data thus suggested that SLT2 was involved in the tolerance of $C$. glabrata to micafungin. SLT2 is part of the so-called fungal cell wall integrity pathway in fungi. Interestingly, SLT2 is also involved in the response of $C$. glabrata to fluconazole, however it is not yet clear if this gene participates to fluconazole tolerance (Schwarzmüller et al., 2014). Echinocandins have been shown to stimulate this pathway in other fungi such as C. albicans (Reinoso-Martín et al., 2003). Mutants lacking this kinase were also shown to be more susceptible in serial dilution assays in C. albicans (LaFayette et al., 2010; Reinoso-Martín et al., 2003). Even though, viability testing were not performed in C. albicans, one may suggest that the cell wall integrity pathway is likely to be involved in echinocandin tolerance as in the case of C. glabrata.

\section{Is antifungal tolerance a favourable ground for development of resistance?}

Resistance to antifungals develops by the acquisition of mutations on specific genes (Cowen et al., 2002; Sanglard and Odds, 2002). Tolerance allows a better survival of fungal pathogens at high drug concentrations and thus the probability to obtain mutations responsible for resistance under drug selective pressure might be higher. There is still no clear evidence in the literature to support this hypothesis. Hsp90 has been reported as important in the process of drug tolerance as above-mentioned (Cowen et al., 2014). Studies have highlighted that Hsp90 is critical for maintaining the capacity of $C$. albicans to develop resistance. Compromising Hsp90 activity in $C$. albicans decreases also the ability of this pathogen to develop, in the presence of azoles, specific inactivating mutations in the gene encoding $\Delta^{5,6}$ sterol desaturase (ERG3) (Cowen and Lindquist, 2005). Even though Hsp90 acts on upstream and downstream elements which control the fate of cells in the presence of other antifungals and growth inhibitors, the role of Hsp90 in the development of resistance has not been yet thoroughly addressed (Cowen, 2013).

\section{Impact of drug tolerance in drug treatment efficacy}

When antifungal susceptibility is measured in vitro and CBP are reached, this leads in most cases to inefficacy of drug treatments in animal experiments. For example, MacCallum et al. (2010) showed that a C. albicans isolate with a fluconazole MIC of $64 \mu \mathrm{g} / \mathrm{ml}$ was not responding to fluconazole treatment in a murine model of invasive infection (Effective dose: $\mathrm{ED}_{50}>200 \mathrm{mg}$ fluconazole $/ \mathrm{kg}$ ). When azole resistance mechanisms related to drug efflux or ERG11 mutations were removed in this isolate, individual progenitors with single resistance mechanisms were obtained and exhibited the same fluconazole MIC $(4 \mu \mathrm{g} / \mathrm{ml})$. The $\mathrm{ED}_{50}$ value for isolates still containing the drug efflux mechanism ( $120 \mathrm{mg}$ fluconazole $/ \mathrm{kg}$ ) was however 10-fold higher than for the ERG11-based mechanism. 
This highlighted that, even if $C$. albicans isolates exhibit similar MIC values, the outcome of therapy depends on the operating resistance mechanism.

The effect of antifungal tolerance on the efficacy of drug treatment is still controversial, given the few available studies. Some studies revealed that the absence of tolerance to a given drug is beneficial for in vivo drug efficacy. For example, when calcineurin activity was inhibited by cyclosporine $A$ in a rat endocarditis infection model with $C$. albicans, fluconazole treatment decreased the fungal load of some infected tissues (heart vegetations) to almost undetectable levels, whereas treatment with fluconazole alone was only moderately decreasing the tissue fungal load (Marchetti et al., 2000a). The beneficial effect of cyclosporine A was due to the fact that fluconazole is fungicidal when calcineurin activity is inhibited. These experiments demonstrated that the inhibition of antifungal tolerance is an interesting concept for the design of more efficient antifungal therapy.

The effect of varying tolerance on in vivo treatment efficacy is less clear. In a first study, Rex et al. (1998) used a set of $C$. albicans isolates with high and low trailing growth characteristics in fluconazole therapy experiments with mice. This study showed that one isolate with trailing growth was responding to fluconazole therapy to the same extent than another isolate without trailing growth. The issue in this study was that the MIC of the trailing isolate at $48 \mathrm{~h}$ reading was above $64 \mu \mathrm{g} / \mathrm{ml}$ (CLSI protocol) while it was $0.25 \mu \mathrm{g} / \mathrm{ml}$ at $24 \mathrm{~h}$ reading. Thus, depending on the mode of endpoint reading, the in vitro susceptibility was not corresponding to treatment outcome. The authors recommended a $24 \mathrm{~h}$ endpoint reading to be coherent with animal data. Noteworthy is a study in which the relationship between caspofungin MICs of several Candida spp. and patient outcomes was investigated. No direct correlation could be observed, thus suggesting that the MIC test method was not adequately reflecting the potential and efficacy of the drug in the patient (Kartsonis et al., 2005). Another work published by Arthington-Skaggs et al. (2000) reached similar conclusions. This study tested two different $C$. albicans isolates with fluconazole trailing characteristics given the divergent endpoint readings at 24 and $48 \mathrm{~h}$ (CLSI protocol). The authors used an alternative sterol quantitation method (SQM) for in vitro determination of azole susceptibility, which measured cellular ergosterol content rather than growth inhibition after exposure to fluconazole. With this method, both trailing isolates exhibited similar SQM MIC values $(\leq 1.0 \mu \mathrm{g} / \mathrm{ml})$. It was interesting to observe that survival of trailing isolates at a fluconazole dosage of $1 \mathrm{mg} / \mathrm{kg} /$ day was not as high as compared to a non-trailing isolate. Second, fungal burden of trailing isolates were significantly higher at day 3 than the other tested non-trailing isolate (Arthington-Skaggs et al., 2000). These data thus suggest that the degree of tolerance (or the degree of trailing growth) can have a significant impact on the kinetics and success of therapy.

\section{Perspectives}

While mediators of antifungal resistance have been well described over the past years, still little attention was given to antifungal tolerance. In this review, we first attempted to clarify the notion of tolerance, which is too often confounded with the description of drug resistance in published studies. We think that antifungal tolerance is important for determining the degree of drug efficacy, however it will be necessary to revisit the relationship between drug tolerance and drug efficacy using appropriate experimental models of infection, adequate dosage and also improved fungal cell detection tools such as live imaging. Moreover, it will be necessary to address whether or not antifungal tolerance can facilitate the development of resistance.
The phenomenon of azole trailing has been described for nearly 2 decades but still no convincing molecular explanation has been provided. In the future, antifungal tolerance should be approached mechanistically with molecular techniques that have been designed recently in the field of fungal pathogens. Not only antifungal tolerance should be addressed in C. albicans but also in non-C. albicans species (for example C. glabrata, $C$. tropicalis, $C$. paraspilosis) as well as in filamentous fungi such as A. fumigatus. The discovery of mediators of antifungal tolerance would be of interest, since they might be targeted by potential inhibitors. This way, they might increase drug efficacy and ultimately may delay the development of resistance.

\section{Acknowledgements}

DS is supported by a grant of the Swiss National Research Foundation 31003A_146936. The authors thank Sara Vaz and Alix Coste for critical reading of the manuscript.

\section{References}

Anderson, T.M., Clay, M.C., Cioffi, A.G., Diaz, K.A., Hisao, G.S., Tuttle, M.D., Nieuwkoop, A.J., Comellas, G., Maryum, N., Wang, S., Uno, B.E., Wildeman, E.L., Gonen, T., Rienstra, C.M., Burke, M.D., 2014. Amphotericin forms an extramembranous and fungicidal sterol sponge. Nat. Chem. Biol. 10, 400-406.

Arendrup, M.C., 2010. Epidemiology of invasive candidiasis. Curr. Opin. Crit. Care 16 445-452.

Arendrup, M.C., Cuenca-Estrella, M., Lass-Florl, C., Hope, W.W., 2013. Breakpoints for antifungal agents: an update from EUCAST focussing on echinocandins against Candida spp. and triazoles against Aspergillus spp. Drug Resist. Updates 16 81-95.

Arendrup, M.C., Perlin, D.S., 2014. Echinocandin resistance: an emerging clinical problem? Curr. Opin. Infect. Dis. $27,484-492$.

Arthington-Skaggs, B.A., Warnock, D.W., Morrison, C.J., 2000. Quantitation of Candida albicans ergosterol content improves the correlation between in vitro antifungal susceptibility test results and in vivo outcome after fluconazole treatment in a murine model of invasive candidiasis. Antimicrob. Agents Chemother. 44, 2081-2085.

Ashley, E.S.D., Lewis, R., Lewis, J.S., Martin, C., Andes, D., 2006. Pharmacology of systemic antifungal agents. Clin. Infect. Dis. S1, S34-S39.

Barchiesi, F., Spreghini, E., Tomassetti, S., Arzeni, D., Giannini, D., Scalise, G., 2005 Comparison of the fungicidal activities of caspofungin and amphotericin B against Candida glabrata. Antimicrob. Agents Chemother. 49, 4989-4992.

Brosh-Nissimov, T., Ben-Ami, R., 2015. Differential association of fluconazole dose and dose/MIC ratio with mortality in patients with Candida albicans and nonalbicans bloodstream infection. Clin. Microbiol. Infect. 21, 1011-1017.

Brown, G.D., Denning, D.W., Gow, N.A., Levitz, S.M., Netea, M.G., White, T.C., 2012 Hidden killers: human fungal infections. Sci. Transl. Med. 4, 165 rv113.

Castanheira, M., Duncanson, F.P., Diekema, D.J., Guarro, J., Jones, R.N., Pfaller, M.A. 2012. Activities of E1210 and comparator agents tested by CLSI and EUCAST broth microdilution methods against Fusarium and Scedosporium species identified using molecular methods. Antimicrob. Agents Chemother. 56, 352-357.

Coste, A., Selmecki, A., Forche, A., Diogo, D., Bougnoux, M.-E., apos, C.D., enfert, J. Berman, D., Sanglard, 2007. Genotypic evolution of azole resistance mechanisms in sequential Candida albicans isolates. Eukaryot. Cell 6, 1889-1904.

Coste, A.T., Crittin, J., Bauser, C., Rohde, B., Sanglard, D., 2009. Functional analysis of cis- and trans-acting elements of the Candida albicans CDR2 promoter with a novel promoter reporter system. Eukaryot. Cell 8, 1250-1267.

Cowen, L.E., 2013. The fungal Achilles' heel: targeting Hsp90 to cripple fungal pathogens. Curr. Opin. Microbiol. 16, 377-384

Cowen, L.E., Anderson, J.B., Kohn, L.M., 2002. Evolution of drug resistance in Candida albicans. Annu. Rev. Microbiol. 56, 139-165.

Cowen, L.E., Lindquist, S., 2005. Hsp90 potentiates the rapid evolution of new traits: drug resistance in diverse fungi. Science 309, 2185-2189.

Cowen, L.E., Sanglard, D., Howard, S.J., Rogers, P.D., Perlin, D.S., 2014. Mechanisms of antifungal drug resistance. Cold Spring Harb. Perspect. Med. 5, a019752.

Denning, D.W., Hope, W.W., 2010. Therapy for fungal diseases: opportunities and priorities. Trends Microbiol. 18, 195-204.

Espinel-Ingroff, A., 2003. In vitro antifungal activities of anidulafungin and micafungin, licensed agents and the investigational triazole posaconazole as determined by NCCLS methods for 12,052 fungal isolates: review of the literature. Rev. Iberoam. Micol. 20, 121-136.

Fiori, A., van Dijck, P., 2012. Potent synergistic effect of doxycycline with fluconazole against Candida albicans is mediated by interference with iron homeostasis Antimicrob. Agents Chemother. 56, 3785-3796.

Fridman, O., Goldberg, A., Ronin, I., Shoresh, N., Balaban, N.Q., 2014. Optimization of lag time underlies antibiotic tolerance in evolved bacterial populations. Nature $513,418-421$. 
Gao, Y., Zhang, C., Lu, C., Liu, P., Li, Y., Li, H., Sun, S., 2013. Synergistic effect of doxycycline and fluconazole against Candida albicans biofilms and the impact of calcium channel blockers. FEMS Yeast Res. 13, 453-462.

Hope, W.W., Drusano, G.L., 2009. Antifungal pharmacokinetics and pharmacodynamics: bridging from the bench to bedside. Clin. Microbiol. Infect. 15, 602-612.

Jacobsen, I.D., Luttich, A., Kurzai, O., Hube, B., Brock, M., 2014. In vivo imaging of disseminated murine Candida albicans infection reveals unexpected host sites of fungal persistence during antifungal therapy. J. Antimicrob. Chemother. 69, 2785-2796.

Kartsonis, N., Killar, J., Mixson, L., Hoe, C.-M., Sable, C., Bartizal, K., Motyl, M., 2005. Caspofungin susceptibility testing of isolates from patients with esophageal candidiasis or invasive candidiasis: relationship of MIC to treatment outcome. Antimicrob. Agents Chemother. 49, 3616-3623.

Kaur, R., Castaño, I., Cormack, B.P., 2004. Functional genomic analysis of fluconazole susceptibility in the pathogenic yeast Candida glabrata: roles of calciun signaling and mitochondria. Antimicrob. Agents Chemother. 48, 1600-1613.

Lackner, M., de Hoog, G.S., Verweij, P.E., Najafzadeh, M.J., Curfs-Breuker, I., Klaassen, C.H., Meis, J.F., 2012. Species-specific antifungal susceptibility patterns of scedosporium and pseudallescheria species. Antimicrob. Agents Chemother. 56 2635-2642.

LaFayette, S.L., Collins, C., Zaas, A.K., Schell, W.A., Betancourt-Quiroz, M., Gunatilaka, A.A., Perfect, J.R., Cowen, L.E., 2010. PKC signaling regulates drug resistance of the fungal pathogen Candida albicans via circuitry comprised of Mkc1, calcineurin and Hsp90. PLoS Pathog. 6, pe1001069.

LaFleur, M.D., Kumamoto, C.A., Lewis, K., 2006. Candida albicans biofilms produce antifungal-tolerant persister cells. Antimicrob. Agents Chemother. 50 3839-3846.

Lepak, A.J., Andes, D.R., 2014. Antifungal Pharmacokinetics and Pharmacodynamics Cold Spring Harb. Perspect. Med. 5, a019653.

Lewis, K., 2007. Persister cells, dormancy and infectious disease. Nat. Rev. Microbiol. $5,48-56$.

Li, P., Seneviratne, C.J., Alpi, E., Vizcaino, J.A., Jin, L., 2015a. Delicate metabolic contro and coordinated stress response critically determine antifungal tolerance of Candida albicans biofilm persisters. Antimicrob. Agents Chemother. 59, 6101-6112.

Li, X., Cai, O., Mei, H., Zhou, X., Shen, Y., Li, D., Liu, W., 2015b. The Rpd3/Hda1 family of histone deacetylases regulates azole resistance in Candida albicans. J. Antimicrob. Chemother. 70, 1993-2003.

Liu, S., Hou, Y., Chen, X., Gao, Y., Li, H., Sun, S., 2014. Combination of fluconazole with non-antifungal agents: a promising approach to cope with resistant Candida albicans infections and insight into new antifungal agent discovery. Int. J Antimicrob. Agents 43, 395-402.

Luna-Tapia, A., Kerns, M.E., Eberle, K.E., Jursic, B.S., Palmer, G.E., 2015. Trafficking through the late endosome significantly impacts Candida albicans tolerance of the azole antifungals. Antimicrob. Agents Chemother. 59, 2410-2420.

MacCallum, D.M., Coste, A., Ischer, F., Jacobsen, M.D., Odds, F.C., Sanglard, D., 2010 Genetic dissection of azole resistance mechanisms in Candida albicans and thei validation in a mouse model of disseminated infection. Antimicrob. Agents Chemother. 54, 1476-1483.

Marchetti, O., Entenza, J.M., Sanglard, D., Bille, J., Glauser, M.P., Moreillon, P., 2000a. Fluconazole plus cyclosporine: a fungicidal combination effective against experimental endocarditis due to Candida albicans. Antimicrob. Agents Chemother. 44, 2932-2938.

Marchetti, O., Moreillon, P., Glauser, M.P., Bille, J., Sanglard, D., 2000b. Potent synergism of the combination of fluconazole and cyclosporine in Candida albicans. Antimicrob. Agents Chemother. 44, 2373-2381.

Marr, K.A., Rustad, T.R., Rex, J.H., White, T.C., 1999. The trailing end point phenotype in antifungal susceptibility testing is $\mathrm{pH}$ dependent. Antimicrob. Agents Chemother. 43, 1383-1386.

Martin, G.S., Mannino, D.M., Eaton, S., Moss, M., 2003. The epidemiology of sepsis in the United States from 1979 through 2000. N. Engl. J. Med. 348, 1546-1554.

Maubon, D., Garnaud, C., Calandra, T., Sanglard, D., Cornet, M., 2014. Resistance of Candida spp. to antifungal drugs in the ICU: where are we now? Intensive Care Med. 40, 1241-1255.
Miyazaki, T., Inamine, T., Yamauchi, S., Nagayoshi, Y., Saijo, T., Izumikawa, K., Seki, M., Kakeya, H., Yamamoto, Y., Yanagihara, K., Miyazaki, Y., Kohno, S., 2010. Role of the Slt2 mitogen-activated protein kinase pathway in cell wall integrity and virulence in Candida glabrata. FEMS Yeast Res. 10, 343-352.

Pfaller, M., Castanheira, M., Diekema, D., Messer, S., Moet, G., Jones, R., 2010. Comparison of European Committee on Antimicrobial Susceptibility Testing (EUCAST) and Etest methods with the CLSI broth microdilution method for echinocandin susceptibility testing of Candida species. J. Clin. Microbiol. 48, $1592-1599$

Pfaller, M.A., 2012. Antifungal drug resistance: mechanisms, epidemiology, and consequences for treatment. Am. J. Med. 125, S3-S13.

Pfaller, M.A., Castanheira, M., Messer, S.A., Rhomberg, P.R., Jones, R.N., 2014. Comparison of EUCAST and CLSI broth microdilution methods for the susceptibility testing of 10 systemically active antifungal agents when tested against Candida spp. Diagn. Microbiol. Infect. Dis. 79, 198-204.

Pfaller, M.A., Messer, S.A., Rhomberg, P.R., Jones, R.N., Castanheira, M., 2013. In vitro activities of isavuconazole and comparator antifungal agents tested against a global collection of opportunistic yeasts and molds. J. Clin. Microbiol. 51, 2608-2616.

Rambach, G., Oberhauser, H., Speth, C., Lass-Florl, C., 2011. Susceptibility of Candida species and various moulds to antimycotic drugs: use of epidemiological cutoff values according to EUCAST and CLSI in an 8-year survey. Med. Mycol. 49, 856-863.

Reinoso-Martín, C., Schüller, C., Schuetzer-Muehlbauer, M., Kuchler, K., 2003. The yeast protein kinase $C$ cell integrity pathway mediates tolerance to the antifungal drug caspofungin through activation of Slt2p mitogen-activated protein kinase signaling. Eukaryot. Cell 2, 1200-1210.

Rex, J., Nelson, P., Paetznick, V., Lozano-Chiu, M., Espinel-Ingroff, A., Anaissie, E., 1998 Optimizing the correlation between results of testing in vitro and therapeutic outcome in vivo for fluconazole by testing critical isolates in a murine model of invasive candidiasis. Antimicrob. Agents Chemother. 42, 129-134.

Richardson, M., Lass-Flörl, C., 2008. Changing epidemiology of systemic fungal infections. Clin. Microbiol. Infect. 14 (Suppl. 4), 5-24.

Robbins, N., Leach, M.D., Cowen, L.E., 2012. Lysine deacetylases Hda1 and Rpd3 regulate Hsp90 function thereby governing fungal drug resistance. Cell Rep. 2, $878-888$.

Sanglard, D., 2011. Diagnosis of antifungal drug resistance mechanisms in fungal pathogens: transcriptional gene regulation. Curr. Fungal Infect. Rep. 5, 157-167, http://dx.doi.org/10.1007/s12281-011-0055-9.

Sanglard, D., Ischer, F., Marchetti, O., Entenza, J., Bille, J., 2003. Calcineurin A of Candida albicans: involvement in antifungal tolerance, cell morphogenesis and virulence. Mol. Microbiol. 48, 959-976.

Sanglard, D., Odds, F.C., 2002. Resistance of Candida species to antifungal agents: molecular mechanisms and clinical consequences. Lancet Infect. Dis. 2, $73-85$.

Schwarzmüller T. Ma B. Hiller, E Istel, F, Tscherner, M., Brunke, S, Ames, L., Firon, A., Green, B., Cabral, V., Marcet-Houben, M., Jacobsen, I.D., Quintin, J., Seider, K., Frohner, I., Glaser, W., Jungwirth, H., Bachellier-Bassi, S., Chauvel, M., Zeidler, U., Ferrandon, D., Gabaldón, T., Hube, B., d'Enfert, C., Rupp, S., Cormack, B., Haynes, K., Kuchler, K., 2014. Systematic phenotyping of a large-scale Candida glabrata deletion collection reveals novel antifungal tolerance genes. PLoS Pathog. 10, e1004211-e1004219.

Tobudic, S., Kratzer, C., Lassnigg, A., Graninger, W., Presterl, E., 2010. In vitro activity of antifungal combinations against Candida albicans biofilms. J. Antimicrob. Chemother. 65, 271-274.

Vasicek, E.M., Berkow, E.L., Flowers, S.A., Barker, K.S., Rogers, P.D., 2014. UPC2 is universally essential for azole antifungal resistance in Candida albicans. Eukaryot. Cell 13, 933-946.

Verstraeten, N., Knapen, W.J., Kint, C.I., Liebens, V., Van den Bergh, B., Dewachter, L., Michiels, J.E., Fu, Q. David, C.C., Fierro, A.C. Marchal, K., Beirlant, J., Versées, W. Hofkens, J., Jansen, M., Fauvart, M., Michiels, J., 2015. Obg and membrane depolarization are part of a microbial bet-hedging strategy that leads to antibiotic tolerance. Mol. Cell 59, 9-21. 